\title{
MONOTONICITY THEOREMS FOR ANALYTIC FUNCTIONS CENTERED AT INFINITY
}

\author{
GALATIA CLEANTHOUS
}

(Communicated by Jeremy Tyson)

\begin{abstract}
We consider the family of analytic functions centered at infinity with Laurent expansion $f(z)=c z+c_{0}+\sum_{j=1}^{\infty} c_{j} z^{-j}$. We prove some monotonicity theorems involving geometric quantities such as diameter, radius and length.
\end{abstract}

\section{INTRODUCTION}

Let us consider the analytic functions $f$ on $\mathbb{C} \backslash \overline{\mathbb{D}}$, where $\mathbb{D}=\{z \in \mathbb{C}:|z|<1\}$, with Laurent expansion at infinity

$$
f(z)=c z+c_{0}+\sum_{j=1}^{\infty} c_{j} z^{-j},
$$

where $c \neq 0$. We denote by $\mathcal{M}$ the family of all the functions of the above form with the usual normalization $c=1$. Let also $\Sigma$ be the subclass of $\mathcal{M}$ which contains the univalent functions in $\mathcal{M}$. The class $\Sigma$ is a classical topic of research; see [4, 7].

For the class $\Sigma$, a well-known result of Pólya and Szegö [11, Problem 141, Page 23 asserts that the diameter $D_{f}$ of the complement of the image of $\mathbb{C} \backslash \overline{\mathbb{D}}$, under $f$, satisfies the inequalities $2 \leq D_{f} \leq 4$. The equality $D_{f}=4$ is attained if and only if $f$ is of the form $f(z)=z+c+e^{i t} / z, c \in \mathbb{C}, t \in \mathbb{R}$. The uniqueness statement of the equality $D_{f}=2$ is that $D_{f}=2$ if and only if $f$ is a translation (i.e. $f(z)=z+c_{0}$, for some constant $c_{0} \in \mathbb{C}$ ). This assertion appeared in 11] but was not proved there. It was proved in [5] by Jenkins. Alternative proofs appeared in [6], [8] and 9].

For every $f \in \mathcal{M}$ and $r>1$ we set $C_{r}=\{z \in \mathbb{C}:|z|=r\}, \Gamma_{f}(r)=f\left(C_{r}\right)$. We denote by $D_{f}(r)$ the diameter of $\Gamma_{f}(r)$ and we prove the following monotonicity theorem:

Theorem 1 (Diameter Theorem). (a) If $f \in \mathcal{M}$, then the function

$$
\varphi_{D}(r)=\frac{D_{f}(r)}{2 r}, \quad 1<r<\infty,
$$

is strictly decreasing, except when $f$ is a translation, in which case $\varphi_{D}$ is constant.

Received by the editors October 19, 2012 and, in revised form, October 24, 2012 and October 29, 2012 .

2010 Mathematics Subject Classification. Primary 30C25, 30C35, 30 C75.

Key words and phrases. Meromorphic functions, conformal mapping, logarithmic capacity, monotonicity theorems, univalent functions, Schwarz Lemma.

The author would like to thank D. Betsakos, her thesis advisor, for his help, and the Cyprus State Scholarship Foundation for its support. 
(b) If $f \in \mathcal{M}$, then $\lim _{r \rightarrow \infty} \varphi_{D}(r)=1$.

(c) If $f \in \Sigma$, then $\lim _{r \rightarrow 1} \varphi_{D}(r)=D_{f} / 2$.

(d) If $f \in \Sigma$, then $D_{f} \geq 2$. Moreover, $D_{f}=2$ if and only if $f$ is a translation.

Similar monotonicity theorems for holomorphic functions $f: \mathbb{D} \rightarrow \mathbb{C}$ have been a subject of interest of several authors. Aulaskari and Chen [1, Theorem 6] prove that the function $\phi_{\text {Area }}(r)=\left(\pi r^{2}\right)^{-1}$ Area $f(r \mathbb{D})$ is increasing for $0<r<1$. Here Area $A$ is the area of the set $A$ and $r \mathbb{D}=\{z \in \mathbb{C}:|z|<r\}$. In [3, Burckel, Marshall, Minda, Poggi-Corradini and Ransford consider the functions

$$
\phi_{\text {Diam }}(r)=\frac{\operatorname{Diam} f(r \mathbb{D})}{2 r}, \phi_{\text {Cap }}(r)=\frac{\operatorname{Cap} f(r \mathbb{D})}{r},
$$

where $\operatorname{Diam} A, \operatorname{Cap} A$ are the diameter and the logarithmic capacity of the set $A$ respectively, and they prove that $\phi_{\text {Diam }}, \phi_{\text {Cap }}$ are strictly increasing for $0<r<1$, except when $f$ is linear (i.e. $f(z)=a z+b$, for some $a, b \in \mathbb{C}$ ), in which case $\phi_{\text {Diam }}, \phi_{\text {Cap }}$ are constant. Moreover, they prove that

$$
\lim _{r \downarrow 0} \phi_{\text {Diam }}(r)=\lim _{r \downarrow 0} \phi_{\text {Cap }}(r)=\left|f^{\prime}(0)\right| \text { and } \lim _{r \downarrow 0} \phi_{\text {Area }}(r)=\left|f^{\prime}(0)\right|^{2} .
$$

In fact, the classical Schwarz Lemma itself can be viewed as a monotonicity theorem (see [3]): We set

$$
\operatorname{Rad} f(r \mathbb{D})=\sup _{z \in r \mathbb{D}}|f(z)-f(0)|, 0<r<1 .
$$

Then the function $\phi_{\operatorname{Rad}}(r)=r^{-1} \operatorname{Rad} f(r \mathbb{D})$ is strictly increasing for $0<r<1$, except when $f$ is linear, in which case $\phi_{\text {Rad }}$ is constant. Moreover, $\lim _{r \downarrow 0} \phi_{\operatorname{Rad}}(r)=$ $\left|f^{\prime}(0)\right|$.

In 2] Betsakos and Pouliasis prove that the function

$$
\Phi_{I}(r)=\frac{R(f(r \mathbb{D}), f(0))}{r},
$$

where $R(D, z)$ is the inner radius of $D$ at $z$, is increasing. For some other related monotonicity theorems, we refer to [2] and the references therein.

For functions $f \in \Sigma$, with Laurent expansion

$$
f(z)=z+c_{0}+\sum_{j=1}^{\infty} c_{j} z^{-j} .
$$

Pólya and Szegö [10, p. 129, Problem 126] (see also [4, p. 29]) prove that the area $A_{f}(r)$ of the domain which is bounded by the Jordan curve $\Gamma_{f}(r)=f\left(C_{r}\right), r>1$, is given by the formula

$$
A_{f}(r)=\pi\left(r^{2}-\sum_{j=1}^{\infty} j\left|c_{j}\right|^{2} r^{-2 j}\right)
$$

and so the function

$$
\varphi_{A}(r)=\left(\pi r^{2}\right)^{-1} A_{f}(r)
$$

equals

$$
\varphi_{A}(r)=1-\sum_{j=1}^{\infty} j\left|c_{j}\right|^{2} r^{-2 j-2} .
$$


Thus $\varphi_{A}$ is strictly increasing unless $c_{j}=0$, for every $j=1,2, \ldots$, in which case $\varphi_{A}$ is constant and $f$ is a translation. Also, by (1.6) we get

$$
\lim _{r \rightarrow \infty} \varphi_{A}(r)=1 \text {. }
$$

The proof of Theorem 1 is in section 2. As we mentioned above, the proof of (d) can also be found in [5, 6, 8, 9]. Some other monotonicity results for functions in $\mathcal{M}$ and $\Sigma$ appear in section 3. These results involve geometric quantities such as radius, length and capacity.

\section{Proof of Theorem 1}

(a) Let $f \in \mathcal{M}$ have the Laurent expansion (1.3). Let $1<r<s$ and $z_{1}, z_{2} \in C_{s}$ be such that $D_{f}(s)=\left|f\left(z_{1}\right)-f\left(z_{2}\right)\right|$. We set $w=z_{1} / z_{2}=e^{i \alpha}, \alpha \in \mathbb{R}$, and we consider the function

$$
\Phi_{s}(z)=f(w z)-f(z), \text { for every } z \in \mathbb{C} \backslash \overline{\mathbb{D}} .
$$

The function $(2 z)^{-1} \Phi_{s}(z)$ is holomorphic in $\mathbb{C} \backslash \overline{\mathbb{D}}$ (including the point at infinity). Also,

$$
\max _{z \in C_{r}}\left|\frac{\Phi_{s}(z)}{2 z}\right| \leq \frac{D_{f}(r)}{2 r} .
$$

Thus, by the maximum principle, for every $z \in \mathbb{C} \backslash r \mathbb{D}$,

$$
\left|\frac{\Phi_{s}(z)}{2 z}\right| \leq \frac{D_{f}(r)}{2 r} .
$$

We set $z=z_{2}$ in (2.2) and by (2.1) we conclude that

$$
\varphi_{D}(s)=\left|\frac{\Phi_{s}\left(z_{2}\right)}{2 z_{2}}\right| \leq \frac{D_{f}(r)}{2 r}=\varphi_{D}(r),
$$

and thus $\varphi_{D}$ is decreasing.

Let us now assume that $\varphi_{D}$ is not strictly decreasing. So there are $1<r<s$ such that $\varphi_{D}(r)=\varphi_{D}(s)$. Then, since $\varphi_{D}$ is decreasing,

$$
\varphi_{D}(r)=\varphi_{D}(\rho)=\varphi_{D}(s) \text {, for every } \rho \in[r, s] .
$$

By the maximum principle, there is a constant $C \in \mathbb{C}$ such that

$$
\frac{\Phi_{s}(z)}{2 z}=C, \text { for every } z \in \mathbb{C} \backslash r \mathbb{D},
$$

or equivalently, using (1.3) and (2.1), we have identically in $\mathbb{C} \backslash r \mathbb{D}$

$$
(w-1) z+\sum_{j=1}^{\infty} c_{j}\left(w^{-j}-1\right) z^{-j}=2 C z,
$$

which means that $w-1=2 C$ and

$$
c_{j}\left(w^{-j}-1\right)=0, \text { for every } j=1,2, \ldots
$$

Since the function $(2 z)^{-1} \Phi_{s}(z)$ is constant, for every $\rho \in[r, s]$ and every $z_{\rho} \in C_{\rho}$,

$$
\left|\frac{\Phi_{s}\left(z_{\rho}\right)}{2 z_{\rho}}\right|=|C|=\varphi_{D}(s)=\varphi_{D}(\rho) .
$$

Thus $D_{f}(\rho)=\left|\Phi_{s}\left(z_{\rho}\right)\right|=\left|f\left(w z_{\rho}\right)-f\left(z_{\rho}\right)\right|$. So the modulus

$$
\left|f\left(\rho e^{i t}\right)-f\left(\rho e^{i \theta}\right)\right|^{2}
$$


is maximized for $t=\alpha+\theta$, for every $\rho \in[r, s]$ and every $\theta \in \mathbb{R}$. Therefore, its partial derivative with respect to $t$ vanishes when $t=\alpha+\theta$, for every $\rho \in[r, s]$. Using the expansion (1.3), we find an expression for the partial derivative for $t=a+\theta$. The coefficients of all the powers of $\rho$ in this expression should be zero. The coefficient of $\rho^{2}$ is

$$
i\left(w^{-1}-w\right)=2 \operatorname{Im}(w) .
$$

Hence $w= \pm 1$. But for $w=1$, we get $z_{1}=z_{2}$, and thus $D_{f}(s)=0$; then $f$ should be constant on the circle $C_{s}$, which gives a contradiction. So $w=-1$. We go back to (2.3) and substitute $w=-1$. Then

$$
c_{2 n+1}=0 \text {, for every } n=0,1, \ldots
$$

Using (2.4) we find that the coefficients of the powers $\rho^{-2 n+1}$ are

$$
-8 n \operatorname{Im}\left(c_{2 n} e^{-i(2 n+1) \theta}\right),
$$

which implies that

$$
c_{2 n}=0, \text { for every } n=1,2, \ldots
$$

We conclude that $c_{j}=0$ for every $j=1,2, \ldots$, and so $f$ is a translation and the proof of (a) is complete.

(b) Let $f \in \mathcal{M}$ have the expansion (1.3). Then the function

$$
g(z)=\frac{1}{f(1 / z)}=\frac{z}{1+c_{0} z+\sum_{j=1}^{\infty} c_{j} z^{1+j}}
$$

is holomorphic in a neighbourhood of $z_{0}=0$. We have that $g(0)=0$ and $g^{\prime}(0)=$ $1 \neq 0$, and so $z_{0}=0$ is a first order zero of $g$. This implies that there exists an $\epsilon>0$ such that $g$ is univalent in the $\operatorname{disc} \epsilon \mathbb{D}$. So the function $f(z)=1 / g(1 / z)$ is univalent in $\mathbb{C} \backslash(1 / \epsilon) \overline{\mathbb{D}}$. Let now $r>1 / \epsilon$. Since $f$ is univalent in $\mathbb{C} \backslash(1 / \epsilon) \overline{\mathbb{D}}, \Gamma_{f}(r)$ is a Jordan curve. We denote by $I_{f}(r)$ the interior of $\Gamma_{f}(r)$ and we set $A_{f}(r)=\operatorname{Area} I_{f}(r)$. The well-known isodiametric inequality asserts that $A_{f}(r) \leq \pi \operatorname{Diam}^{2} I_{f}(r) / 4$, and hence, by (1.5),

$$
\sqrt{\varphi_{A}(r)} \leq \varphi_{D}(r)
$$

By (1.3), one easily obtains that

$$
\varphi_{D}(r) \leq 1+\sum_{j=1}^{\infty}\left|c_{j}\right| r^{-j-1} .
$$

By (2.6), (2.7) and (1.7), we get

$$
\lim _{r \rightarrow \infty} \varphi_{D}(r)=1 .
$$

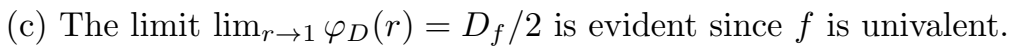

(d) Let $f \in \Sigma$ and $r>1$. By (a), (b) and (c) we have that

$$
1=\lim _{\rho \rightarrow \infty} \varphi_{D}(\rho) \leq \varphi_{D}(r) \leq \lim _{\rho \rightarrow 1} \varphi_{D}(\rho)=\frac{D_{f}}{2},
$$

and so $D_{f} \geq 2$. The equality $D_{f}=2$ now holds if and only if $\varphi_{D}$ is constant and so if and only if $f$ is a translation. 


\section{RADIUS, LENGTH AND CAPACITY THEOREMS}

Suppose $f \in \mathcal{M}$ has the expansion (1.3). We introduce a kind of radius. We cannot use (1.2) since $f(0)$ is not at our disposal. We substitute it with the constant term $c_{0}$ of the Laurent expansion, which is called the conformal center of the complement of $f(\mathbb{C} \backslash \overline{\mathbb{D}})$; see [11, p. 23], [7, p. 12]. We thus set

$$
R_{f}(r)=\sup _{z \in C_{r}}\left|f(z)-c_{0}\right|, \quad r>1 .
$$

Theorem 2 (Radius Theorem). Let $f \in \mathcal{M}$. The function $\varphi_{R}(r)=r^{-1} R_{f}(r)$ is strictly decreasing for $r>1$, except when $f$ is a translation, in which case $\varphi_{R}$ is constant. Moreover, $\lim _{r \rightarrow \infty} \varphi_{R}(r)=1$.

Proof. Let $f \in \mathcal{M}$ with the expansion (1.3). Then $f(z)-c_{0}=z+\sum_{j=1}^{\infty} c_{j} z^{-j}$. Let $1<r<s$. We have that

$$
\begin{aligned}
\varphi_{R}(r) & =\frac{\sup _{z \in C_{r}}\left|z+\sum_{j=1}^{\infty} c_{j} z^{-j}\right|}{r} \\
& =\sup _{z \in C_{r}}\left|1+\sum_{j=1}^{\infty} c_{j} z^{-j-1}\right| .
\end{aligned}
$$

We apply the maximum principle to the function

$$
h(\zeta)=1+\sum_{j=1}^{\infty} c_{j} \zeta^{j+1}, \zeta \in \mathbb{D},
$$

and we conclude that $\varphi_{R}$ is strictly decreasing unless $f$ is a translation. Moreover, it follows from (3.1) that $\lim _{r \rightarrow \infty} \varphi_{R}(r)=1$.

We now present an analogous monotonicity result involving length. For $f \in \Sigma$, $\Gamma_{f}(r)$ is a smooth Jordan curve. Let $L_{f}(r)$ be its length.

Theorem 3 (Length Theorem). Let $f \in \Sigma$. The function $\varphi_{L}(r)=(2 \pi r)^{-1} L_{f}(r)$ is strictly decreasing for $r>1$, except when $f$ is a translation, in which case $\varphi_{L}$ is constant. Moreover, $\lim _{r \rightarrow \infty} \varphi_{L}(r)=1$.

Proof. Let $f \in \Sigma$. The function $g(z)=\left(f^{\prime}(z)\right)^{1 / 2}$ has the Laurent expansion at the point of infinity

$$
g(z)=1+\sum_{j=2}^{\infty} b_{j} z^{-j} .
$$

Let $r>1$. As we mentioned above, the curve $\Gamma_{f}(r)$ is a smooth Jordan curve and has the parametrization $\gamma_{r}(t)=f\left(r e^{i t}\right), t \in[0,2 \pi]$. So its length is

$$
L_{f}(r)=\int_{0}^{2 \pi}\left|\gamma_{r}^{\prime}(t)\right| d t=r \int_{0}^{2 \pi}\left|g\left(r e^{i t}\right)\right|^{2} d t .
$$

After standard calculations involving the power series (3.2), we find that

$$
\int_{0}^{2 \pi}\left|g\left(r e^{i t}\right)\right|^{2} d t=2 \pi+2 \pi \sum_{j=2}^{\infty}\left|b_{j}\right|^{2} r^{-2 j} .
$$


Combining the relations (3.3) and (3.4), we obtain

$$
\varphi_{L}(r)=1+\sum_{j=2}^{\infty}\left|b_{j}\right|^{2} r^{-2 j},
$$

and so $\varphi_{L}$ is strictly decreasing unless $b_{j}=0$, for every $j=2,3, \ldots$, in which case $\varphi_{L}$ is constant. In this case, because of (3.2), we have $f^{\prime}(z)=1$, which means exactly that $f$ is a translation. Also, by (3.5), we obtain that $\lim _{r \rightarrow \infty} \varphi_{L}(r)=1$.

We give via conformal mapping [7 the definition of logarithmic capacity of a compact set $E \subset \mathbb{C}$, and we denote it by $\operatorname{Cap} E$. For other definitions (via transfinite diameter and equilibrium measure) we refer the reader to [12].

Let $E \subset \mathbb{C}$, be a simply connected compact set. Let

$$
f(z)=a_{1} z+a_{0}+\sum_{j=1}^{\infty} a_{-j} z^{-j}
$$

be the unique conformal mapping from $\mathbb{C} \backslash \overline{\mathbb{D}}$ onto $\mathbb{C} \backslash E$ with $a_{1}>0$, which maps $\infty$ to $\infty$. Then the logarithmic capacity of the set $E$ is defined as $\operatorname{Cap} E=a_{1}$.

Now for every $f \in \Sigma$ and every $r>1$ we set $C_{f}(r)=\operatorname{Cap} I_{f}(r)$. The following theorem follows directly from the above definition of logarithmic capacity.

Theorem 4 (Capacity Theorem). Let $f \in \Sigma$. The function $\varphi_{C}(r)=r^{-1} C_{f}(r)$ is constant and equals 1 for every $r>1$.

\section{FinAL REMARKS}

In [3] the authors study the so-called higher-order diameters, which are defined for a set $E \subset \mathbb{C}$ as

$$
d_{n}(E)=\sup \left(\prod_{j<k}\left|z_{j}-z_{k}\right|\right)^{2 /(n(n-1))},
$$

where the supremum is taken over all $n$-tuples of points from $E$.

There are two limit cases here:

(a) When $n=2$, we have that $d_{2}(E)=\operatorname{Diam}(E)$ and

(b) when $n=\infty$, we have that $d_{\infty}(E):=\lim _{n \rightarrow \infty} d_{n}(E)=\operatorname{Cap}(E)$.

For the last see also [12, p. 153]. Also it is well-known that $d_{n}(\mathbb{D})=n^{1 /(n-1)}$, and so $\operatorname{Cap}(\mathbb{D})=d_{\infty}(\mathbb{D})=1$. In 3 the authors proved that the functions

$$
\phi_{\text {n-Diam }}(r)=\frac{d_{n}(f(r \mathbb{D}))}{d_{n}(r \mathbb{D})} \text { and } \phi_{\text {Cap }}(r)=\frac{\operatorname{Cap}(f(r \mathbb{D}))}{\operatorname{Cap}(r \mathbb{D})}=\frac{d_{\infty}(f(r \mathbb{D}))}{d_{\infty}(r \mathbb{D})}
$$

are increasing. In our situation we give the following open problem:

Conjecture 1. If $f \in \mathcal{M}$ and $n=2,3, \ldots$, then the function

$$
\varphi_{\mathrm{n}-\operatorname{Diam}}(r):=\frac{d_{n}\left(f\left(C_{r}\right)\right)}{d_{n}\left(C_{r}\right)}, 1<r<\infty,
$$

is strictly decreasing, except when $f$ is a translation, in which case $\varphi_{\mathrm{n}-\operatorname{Diam}}(r)$ is constant. Moreover, $\lim _{r \rightarrow \infty} \varphi_{\mathrm{n}-\operatorname{Diam}}(r)=1$.

As we already mentioned for $n=2$ we have the case of diameter, and so Theorem 1 covers this conjecture. Note also that, in the class $\Sigma$, the limit-case function $\varphi_{C}(r)$ is constant, as one may see in Theorem 4. 


\section{REFERENCES}

[1] Rauno Aulaskari and Huaihui Chen, Area inequality and $Q_{p}$ norm, J. Funct. Anal. 221 (2005), no. 1, 1-24, DOI 10.1016/j.jfa.2004.12.007. MR2124895 (2005k:30066)

[2] Dimitrios Betsakos and Stamatis Pouliasis, Versions of Schwarz's Lemma for Condenser Capacity and Inner Radius, Canad. Math. Bull. 56 (2013), no. 2, 241-250, DOI 10.4153/CMB2011-189-8. MR.3043051

[3] Robert B. Burckel, Donald E. Marshall, David Minda, Pietro Poggi-Corradini, and Thomas J. Ransford, Area, capacity and diameter versions of Schwarz's lemma, Conform. Geom. Dyn. 12 (2008), 133-152, DOI 10.1090/S1088-4173-08-00181-1. MR2434356 (2010j:30050)

[4] Peter L. Duren, Univalent functions, Grundlehren der Mathematischen Wissenschaften [Fundamental Principles of Mathematical Sciences], vol. 259, Springer-Verlag, New York, 1983. MR708494 (85j:30034)

[5] James A. Jenkins, A uniqueness result in conformal mapping, Proc. Amer. Math. Soc. 22 (1969), 324-325. MR0241619 (39 \#2958)

[6] James A. Jenkins, A uniqueness result in conformal mapping. II, Proc. Amer. Math. Soc. 85 (1982), no. 2, 231-232, DOI 10.2307/2044287. MR652448(83h:30020)

[7] Christian Pommerenke, Univalent functions, with a chapter on quadratic differentials by Gerd Jensen. Studia Mathematica/Mathematische Lehrbücher, Band XXV, Vandenhoeck \& Ruprecht, Göttingen, 1975. MR0507768 (58 \#22526)

[8] Albert Pfluger, On a uniqueness theorem in conformal mapping, Michigan Math. J. 23 (1976), no. 4, 363-365 (1977). MR0442207 (56 \#593)

[9] Albert Pfluger, On the diameter of planar curves and Fourier coefficients (English, with German summary), Z. Angew. Math. Phys. 30 (1979), no. 2, 305-314, DOI 10.1007/BF01601942. MR.535988 (81b:42024)

[10] G. Pólya and G. Szegö, Problems and Theorems in Analysis. Vol. I, Springer-Verlag, Berlin, 1972 (first edition in German, 1925). MR0344042

[11] G. Pólya and G. Szegö, Problems and theorems in analysis. Vol. II, Theory of functions, zeros, polynomials, determinants, number theory, geometry. Revised and enlarged translation by C. E. Billigheimer of the fourth German edition, Die Grundlehren der Mathematischen Wissenschaften, Band 216, Springer-Verlag, New York, 1976. MR.0396134 (53 \#2)

[12] Thomas Ransford, Potential theory in the complex plane, London Mathematical Society Student Texts, vol. 28, Cambridge University Press, Cambridge, 1995. MR.1334766 (96e:31001)

Department of Mathematics, Aristotle University of Thessaloniki, Thessaloniki 54124, GREECE

E-mail address: gkleanth@math.auth.gr 